\title{
THE DENGUE VACCINES: ASSESSMENT OF FUTURE PROSPECTS, TREATMENT, AND VACCINE CHALLENGES
}

\author{
GADE KALYANI ${ }^{1,2 *}$, THANUSHREE $\mathbf{N}^{3}$ \\ ${ }^{1}$ Department of Pharmacology, Gautham College of Pharmacy, Bengaluru, Karnataka, India. ${ }^{2}$ Department of Pharmaceutical Chemistry, \\ University College of Pharmaceutical Sciences, Acharya Nagarjuna University, Guntur, Andhra Pradesh, India. ${ }^{3}$ Department of Pharmacy \\ Practice, Gautham College of Pharmacy, Bengaluru, Karnataka, India. Email: gadekalyani1989@gmail.com
}

Received: 06 March 2020, Revised and Accepted: 09 April 2020

\section{ABSTRACT}

Dengue virus (DENV) is the fastest re-emerging arbovirus existing in the tropical and subtropical regions today. It has become a worldwide major public health problem, especially affecting South-East Asian populations. Dengue is spread by Aedes mosquito bite. It has three phases based on severity termed as febrile, critical and recovery phases. As per the national survey data, the prevalence of dengue has been still rising consistently, and the burden of the disease is predominantly affecting the adults with progression towards the Dengue fever (DF), Dengue hemorrhagic fever (DHF) and Dengue shock syndrome (DSS). The key elements needed to achieve the dengue public health targets identified by the World health organization (WHO) global strategy are the diagnosis, care management, outbreak preparedness and integrated surveillance, sustainable vector control, future vaccine implementation. This review mainly describes the dengue treatment challenges, in addition to a brief discussion of dengue vaccine challenges and future outlook on dengue itself.

Keywords: Dengue virus, Crystalloid fluid, Antibody-dependent enhancement, Attenuated vaccine, Serotype, Hematocrit levels.

(C) 2020 The Authors. Published by Innovare Academic Sciences Pvt Ltd. This is an open access article under the CC BY license (http://creativecommons. org/licenses/by/4. 0/) DOI: http://dx.doi.org/10.22159/ajpcr.2020.v13i6.37366

\section{INTRODUCTION}

Dengue is caused by dengue virus (DENV), a mosquito-borne Flavivirus [1]. Dengue ribonucleic acid (RNA) virus can be classified into five serotypes DENV-1, DENV-2, DENV-3, and DENV-4 and DENV-5 belonging to Flaviviridae family. DENV causes a wide range of diseases in humans, from a self-limited dengue fever (DF) to a life-threatening syndrome called dengue hemorrhagic fever (DHF) or dengue shock syndrome (DSS) [2]. The genome is transcribed as a single open reading frame encoding with three structural (C, PrM, and E) and seven non-structural (NS1, NS2A, NS3, NS4A, NS4B, and NS5) proteins [3]. Each DENV serotype is phylogenetically distinct, suggesting that each stereotype could be considered a different virus [4]. The transmission cycle is "man-mosquito-man" [5,6]. The symptoms of dengue include a high fever with chills, rashes, and severe headaches. The two hallmarks of DHF are leakage of plasma and that can be led to hypovolemic shock and abnormal hemostasis $[7,8]$.

\section{DENGUE TREATMENT CHALLENGE}

DF management is supportive and symptomatic [9-11]. During the acute febrile phase of the illness, antipyretics and bed rest are sufficient. In DHF, aspirin should be avoided, as it is known to trigger hemorrhagic manifestations [12]. For patients with vomiting and diarrhea, excessive sweating, oral fluids, and electrolyte therapy are recommended $[13,14]$. The management of DHF during the febrile phase is similar to that of DF [15]. Usually, significant plasma loss is seen in dengue patients, it leads to a rise in hematocrit value, to overcome this, parenteral fluid therapy is recommended $[16,17]$. Hospitalization is recommended in patients with any signs of bleeding and persistent high hematocrit. The colloidal (dextran 40 and plasma) and crystalloid (5\% dextrose in lactated Ringer's solution) are types of fluids used for volume replacement $[18,19]$. The volume of a fluid replacement should be titrated according to vital signs and should be kept to a minimum, but sufficient to maintain effective circulation [20].

For rapid volume replacement, initially crystalloid fluid is used; however, colloidal fluid is indicated in the case of massive leakage and to whom a large volume of crystalloid fluid has been given. Blood transfusion is indicated in certain cases, with profound or persistent shock despite falling hematocrit values after initial fluid replacement. For severe thrombocytopenia and hemodynamically stable patient's prophylactic platelet transfusions are indicated [21,22]. Platelet transfusions have not been shown effective and hence are not required [23]. There are no antiviral agents for the treatment of dengue infection in the present situation. Intravenous rehydration is the therapy of choice. The dynamic nature of dengue demands close monitoring and repeated clinical and laboratory evaluations. The best result is obtained from fresh frozen plasma and cryoprecipitate transfusions to the platelets.

The febrile phase is symptomatic in the management of DF. Therapy for a fever is managed with paracetamol $[24,25]$. Salicylates and other nonsteroidal anti-inflammatory drugs should be avoided as they may cause mucosal bleeding in patients [26]. The patient should be hospitalized who develop cold extremities, restlessness, acute abdominal pain, decreased urine output, bleeding, and hemoconcentration. Children with rising hematocrit levels and thrombocytopenia without clinical symptoms require hospitalization. Early detection of these conditions is important for the reduction of case fatality rates (CDRs) [27]. The most important element of treatment in a patient with DSS is providing intense care with close urinary output, hemorrhagic manifestations, and level of consciousness with adequate and appropriate fluid replacement $[28,29]$.

\section{DENGUE VACCINE}

The first dengue vaccine was discovered in 1929 with mixed results. A lot of challenges are involved in developing effective and safe dengue vaccine [30]. An ideal vaccine for dengue should provide a lifelong protective immune response that neutralizes antibodies that are almost replicating all the four serotypes of (DENV-1-4) [31].

\section{(a) Live attenuated dengue vaccine}

These vaccines have the strength to stimulate robust immune responses and immune memory very similar to that induced during natural 
infection by wild type viruses. These vaccines should be tetravalent formulations that produce a balanced neutralizing immune response that protects against all four serotypes. Live attenuated vaccines for dengue using mutagenesis have developed in the United States, the National Institute of Health in collaboration with Johns Hopkins University [32]. These vaccines are created besides by attenuating mutations into the genomes of each virus in the 31 untranslated regions. Another live-attenuated dengue vaccine that has entered the clinic was developed by the Walter Reed Army Institute of Research (WRAIR) in partnership with Glaxo Smith - Kline (GSK) [33]. To discover a live DENV vaccine, it must be guided with some principles like vaccine should be non-reactogenic and safe, the vaccine strains need to show limited replication in humans to preclude the development of clinically symptomatic dengue infection in vaccinated individuals [34]. Dengue serotype 1, 2, and 4 viruses were developed initially in dog kidney cells, whereas dengue serotype 3 was serially passaged in primary African green monkey kidney cells [35].

\section{(b) Purified inactivated dengue vaccine}

A tetravalent vaccine using purified inactivated versions of the virus is pursued by WRAIR/GSK partnership [36]. By growing the virus in a VERO cell line, these viruses are (epithelial cells of African Green Monkey) followed by formalin with Alum or a proprietary GSK adjustment $[37,38]$. This vaccine was shown to be immunogenic and protective in mice and rhesus macaques [39-43]. The protection of the animals against the challenge with a homologous wild DENV is done by three doses of inactivated vaccine. An inactivated dengue vaccine has been tested as the priming vaccine in a prime-boost immunization strategy, with a live attenuated vaccine as a booster, leading to complete protection in macaques.

\section{(c) Recombinant protein dengue vaccine [37]}

By Merck and co, the recombinant dengue vaccine to DENV-1 is discovered. In various expression systems like Escherichia coli, viral vectors like baculovirus in insect cells, drosophila, and vaccinia virus in yeast cells, recombinant $\mathrm{E}$ protein has been produced. The protein antigen in this vaccine, HBV-001 D1, is a recombinant truncated form of the E protein of the virus and is expressed using a Drosophila system.

\section{(d) Dengue deoxyribose nucleic acid (DNA) vaccine}

By using the virus's PrM and E proteins as the primary targets on a DNA platform, the vaccine is pursued by the U.S Naval medical research center $[37,44]$. By cloning PrM and E of each serotype into plasmid vectors naval medical research center, WRAIR, Maryland, USA has discovered a candidate dengue DNA vaccine. This vaccine was having the capacity to mount neutralizing antibody response and partial protection against different DENV serotypes in mice and Rhesus macaques [45-47].

\section{(e) Chimeric live-attenuated dengue vaccine}

In the initiative of developing a DENV vaccine, Sanofi Pasteur is the furthest one currently [48-55]. The centers for prevention and disease control in the United States have also developed a chimeric dengue vaccine, DENV-axe, which has been licensed to Inviragen, Inc. This particular vaccine uses attenuated DENV-2 backbone E genes replaced with those of the other serotypes of dengue. In May 2019, Dengavaxia was approved by the U.S and Food and Drug Administration in the United States for use in children 9-16-year-old living in the U.S territories of Puerto, American Samoa, the U.S Virgin Islands, and Rico. Different strategies in dengue vaccine development were discussed in Fig. 1.

\section{DENGUE VACCINE CHALLENGES}

A safe and effective dengue vaccine should have the capacity to provide a life-long protective immune response in the form of neutralizing antibodies that should be equally against all types of serotypes [56]. The dengue vaccine field is robust with numerous candidates in preclinical and clinical development [57]. Dengue vaccine remains a precious strategy in the transmission of DENV [58]. Antibodydependent enhancement (ADE) or immune enhancement is observed in DENV infection due to heterologous non-neutralizing antibodies and this makes the potential risk of precipitating serious manifestations such as DHF or DSS during secondary infection by a different serotype. Primary infection with one serotype of the virus provides maximum protective immunity to that homologous serotype. This is mediated by serotype-specific neutralizing antibodies that block viral attachment, prevent fusion of the virus membrane with endocytic vacuole membrane, and inhibit the release of viral RNA into the cytoplasm and promote complement-mediated lysis of antibody-coated virus. Preexisting non-neutralizing heterotypic antibodies from a previous DENV infection allows the virus during a subsequent heterotypic infection, to bind to surface-expressed Fc gamma receptors on monocytes and macrophages.

During the brief period of viremia post-immunization, the live attenuated dengue vaccine is linked with a hypothetical risk of ADE. Due to increased levels of multiplication, due to the attenuated phenotype of the vaccine strains, the risk of vaccine virus causing severe disease. The lack of an appropriate animal model that elucidates the pathogenesis, immune response, and clinical course of dengue infections in humans one of the major difficulties is associated with the development of a safe and effective dengue vaccine. Dengue vaccines have also been challenged by critical issues such as the absence of suitable markers of protective immunity and lack animal models for the disease [59].

According to the directorate of national vector-borne disease control program, India has shown an increasing trend in the number of reported dengue cases, which are almost increased from 5534 in 2007 to 75,454 in 2013 in the past decade, the CFR has decreased from 3.3\% in 1996 to $0.4 \%$ in 2010 with improvement in the surveillance system and effective management. Due to repeated dengue outbreaks, a financial burden to the health care system is increasing rapidly. This gives a clear structure to the need for a cost-effective dengue vaccine as a preventive strategy along with vector control [37].

The capable of monitoring the circulating DENV serotypes in different regions is by the best community based, laboratory-based, and hospitalbased surveillance system and is difficult for vaccine efficacy in the trial. The capacity to detect all febrile illnesses due to dengue in the community is by an active surveillance system, both mild and severe, is also necessary for addition to vaccine efficacy. This leads to becoming a challenge, especially in resource-limited suburban and rural areas. To address the issue of cost-effective implementation, an economic analysis of any possible dengue vaccination strategy is required to be carried out in India for the acceptable cost of a new vaccine for usage in the public sector that may vary from one country to another. A multiple-dose regime, required for balanced immune response in dengue vaccination, can be difficult to implement in India, because of the significant drop-out rates [37].

\section{DENGUE PROSPECTS [60]}

Due to geographical expansion and incidence of dengue, cases will increase in upcoming days and this is according to many experts of the World Health Organization (WHO) [61]. It is therefore required to elaborate on some of the potential factors that drive dengue activity, as well as the global strategic direction to address.

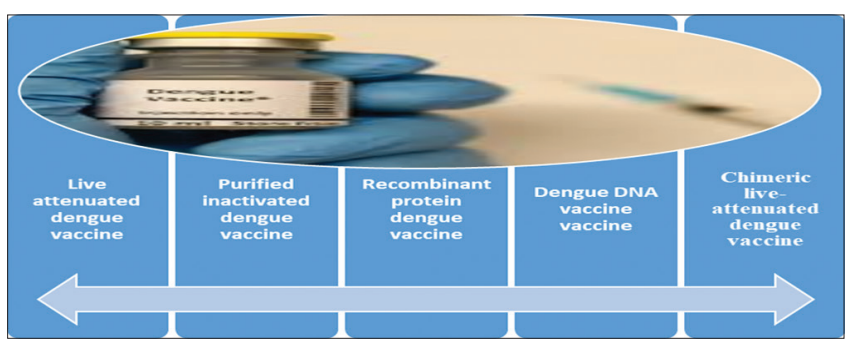

Fig. 1: Chart showing the strategies associated with dengue vaccine development 


\section{POTENTIAL FACTORS THAT DRIVE DENGUE ACTIVITY}

\section{Climate change factors}

The temperature has a prominent role in viral replication, adult vector survival, and infective periods [62,63]. Increased survival and/or migration of vectors into previously non-endemic geographic areas outside the tropics have resulted from increased temperature. Weather variability may enhance environmental and climatic conditions conducive to the proliferation of Aedes species in regions that are currently non-endemic. Climate has both direct effects on dengue incidence and indirect effects mediated by mosquito density, as mosquitoes are the vectors of dengue [64]. Temperature, rainfall, and relative humidity are thought of as important climatic factors contributing toward the growth and dispersion of the mosquito vector [65].

A possible increase in dengue transmission due to higher temperatures, humidity, and precipitation is associated with changes in climate and this is based on some studies of climate change and dengue. This indicates the situation that observed climatic changes, including increased humidity and increased intermediate global temperature which theoretically may increase the epidemic potential of dengue [66]. The individual role climate change plays in the past decade's resurgence of dengue stays uncertain and is a region of current modeling research.

\section{Globalization, travel, and trade factors}

For the current and ongoing expansion of dengue, only climate change is not sufficient. For the rapid expansion of the vector-borne communicable disease, modern contributing factors include globalization factors such as travel and trade associated with vector accommodating trends in modern human settlement and suitable climate conditions $[67,68]$. To describe the recent increase in dengue transmission and mobility, both vector and human populations are the most important variables [69].

Human settlement factors and climate explains the risk of introduction or reintroduction of dengue into non-endemic zones leading to endemic transmission [70]. The main driver of global transmission and expansion of the disease is thought of as an infected dengue person who will be involved in traveling. Globalization factors, which are contributing to the expansion of dengue transmission and the risk of importation of dengue, include trade and travel. Dengue's primary and secondary vectors can also export and import by International transport of cargo and goods, especially through commercial sea shipment. In conclusion, the globalization of humans and mosquitoes is playing an important role in the reemergence of dengue [71].

\section{Settlement factors}

Trends in current human settlement, together with rapidly expanded urban areas, exploding population density and limited socioeconomic resources, suggest that the human factors in addition to climate factors. To suggest that human populations and their collective actions strongly contributes to the transmission of dengue settlement and for this human and socioeconomic factor combine with climate suitably and globalized travel and trade.

Successive contributing factors to the increase of global dengue transmission and geographic expansions are considered by rapid urbanization and population growth [72-74]. The creation of urban breeding sites for the most potent dengue vector Aedes aegypti is done by rapid urbanization. The raised density of both mosquito and human populations as part of urban population growth compounds this effect in terms of both vector suitability and transmission of dengue. Rapid urbanization has provided appropriate circumstances for vector breeding and the high population density has also increased contact frequency between humans and mosquito vectors [75].

\section{Global strategic direction}

It was essential to reflect on policy and strategic direction that attempts to reduce the impact of this disease is by the presence of potential for ceaseless expansion of dengue globally. Based on the historical lack of coordinated efforts, political will and research attention despite the significant diseases, social and economic burden it places intentionally, by this dengue, has been considered as a neglected tropical disease. To decrease the burden of dengue is the overall aim of this multi-sectoral strategy. In response to the sharp increase in the global burden caused by DENV over the past few decades, the WHO has set out three specific key objectives in its disease control strategy: (i) To estimate the true burden of dengue by 2015; (ii) a reduction in dengue mortality by at least $50 \%$ by 2020 (used as a baseline); and (iii) a reduction in dengue morbidity by at least $25 \%$ by 2020 [76-78].

Integrated vector management (IVM) is a global strategic framework first adopted in 2004 by the WHO for all vector-borne diseases [79]. IVM is the key measure for dengue control $[80,81]$. It is a strategy that aims to achieve a maximum impact on vector-borne diseases [82]. Integrated Vector Management (IVM) is the strategic approach for dengue control as promoted by WHO [80]. IVM helps in controlling most of the mosquito vector-borne diseases [81]. This method aims at improving efficacy, sustainability, and cost effectiveness of vector control [82-89]. The reduction of dengue transmission and the resulting disease burden can be done by utilizing an effective integrated vector control strategy. An IVM approach requires entomological knowledge, technical and infrastructure capacity, and systems facilitating stakeholder collaboration [90]. New larval control strategies for IVM of A. aegypti are in high demand, including the use of biological control agents [91].

\section{Role of natural agents in dengue vector control}

Natural chemicals have considerable potential for vector management because these chemicals are safer than conventional insecticides on account of their rapid environmental biodegradation and low toxicity to natural enemies, humans, and other mammals and they suffer less from problems of registration difficulties [92]. IVM understands the local ecosystem, conducts regular field observations and helps in reduction of pests/vectors by implementing changes in ecological, economic, biological and chemical methods that are used for controlling vectors [93]. The species Piper nigrum, Piper aduncum, and Piper longum, may be a feasible alternative source of larvicidal metabolites to control dengue mosquitoes [94]. Lamiaceae and Zingiberaceae plant extracts act as natural larvicidal agent [95].

The ethanolic extracts of seaweed of Dactyladenia dichotoma possess active compounds for the development of larvicidal activity [96]. The methanol extract of Taiwanese seaweed Gracilaria firma is more effective and is an ideal eco-friendly approach for the control larvae of the dengue vector $A$. aegypti [97]. Spinosad is likely to be an effective larvicide for the treatment of mosquito breeding sites [98]. Both the leaf extract of Aloe vera and bio-control agent Bacillus sphaericus could serve as potential larvicidal agents against the dengue vector A. aegypti [99]. The combined effect of the mosquitocidal activity of Solanum xanthocarpum fruit extract and copepods Mesocyclops thermocyclopoides used against the dengue vector [100]. Copepods are very useful in biological control of A. aegypti and Aedes albopictus but also successful to manage the populations of Culex and Anopheles [101]. Argemone mexicana acts as a larvicide against the dengue vector [102]. Microbial pesticides obtained from actinomycetes, Bacillus thuringiensis (Bt), and many other microorganisms are used as eco-friendly alternatives for mosquito control [103].

\section{DISCUSSION}

Dengue is endemic throughout the tropical world. We discussed a treatment strategy to treat dengue infection in various stages of DF. A lot of challenges are faced by developers to discover a safe and effective dengue vaccine. Globalization, trade, travel, and climate change are some of the potential factors responsible for dengue activity. The role of natural chemicals is also discussed which helps in reducing vector control. 


\section{CONCLUSION}

Dengue is a global health problem in the world's population which causes severe illness and rarely leads to the life-threatening syndrome. There is no specific treatment for dengue and the management is only supportive. A safe and effective dengue vaccine gives a lifelong protective immune response and it is equally against all serotypes of dengue. The use of natural agents can prevent dengue vector control in various stages of vector development.

\section{ACKNOWLEDGMENTS}

This review article would not have been possible without the exceptional support of my Ph.D. supervisor Dr. D. Ravi Sankar Reddy, and my husband Raj, who actively inspired me throughout the drafting of this paper and the principal, University College of pharmaceutical sciences, is gratefully acknowledged. We are also thankful for Dr. Rinkumathappan, principal of Gautham College of Pharmacy, Bengaluru, for encouraging us in writing this review article.

\section{AUTHORS' CONTRIBUTIONS}

Gade. Kalyani contributed to intellectualizing the ideas for this review article and Thanushree worked for writing the paper from the ideas obtained from distinct sources. Dr. D. Ravi Sankar reddy helped supervise the review article and Dr. Rinku Mathappan provided critical feedback. The corresponding author and the co-author helped in shaping this manuscript.

\section{CONFLICTS OF INTEREST}

The authors of this review article declare that we have no conflicts of interest.

\section{AUTHORS' FUNDING}

We, the authors of this review article, declare that we do not have any funding sources.

\section{REFERENCES}

1. Jennifer BP. Dengue fever and dengue haemorrhagic fever book review. Nurs J India 2006;11:246-7.

2. Tayade T. Emerging therapy for dengue. Int $\mathrm{J}$ Curr Pharm Res 2018;10:1-4

3. Ayukekbong JA. Dengue virus in Nigeria: Current status and future perspective. Br J Virol 2014;1:106-111.

4. Nedjadi T, El-Kafrawy S, Sohrab SS, Desprès P, Damanhouri G, Azhar E. Tackling dengue fever: Current status and challenges. Virol J 2015;12:212.

5. Brady OJ, Gething PW, Bhatt S, Messina JP, Brownstein JS, Hoen AG, et al. Refining the global spatial limits of dengue virus transmission by evidence-based consensus. PLoS Negl Trop Dis 2012;6:e1760.

6. Chotigeat UR, Kalayanarooj S, Nisalak A. Vertical transmission of dengue infection in Thai infants: Two case reports. Med Assoc Thai 2003;86 Suppl 3:628-32.

7. Raafat N, Blacksell SD, Maude RJ. A review of dengue diagnostics and implications for surveillance and control. Trans R Soc Trop Hyg 2019;113:653-60.

8. Srivastava VK, Suri S, Bhasin A, Srivastava L, Bharadwaj M. An epidemic of dengue haemorrhagic fever and dengue shock syndrome in Delhi: A clinical study. Ann Trop Paediatr 1990;10:329-34.

9. Soni A, Chugh K, Sachdev A, Gupta D. Management of dengue fever in ICU. Indian J Pediatr 2001;68:1051-5.

10. Wiwanitkit V. Dengue fever: Diagnosis and treatment. Expert Rev Anti Infect Ther 2010;8:841-5.

11. Da Fonseca BA, Fonseca SN. Dengue virus infections. Curr Opin Pediatr 2002;14:67-71.

12. Jahan F. Dengue fever (DF) in Pakistan. Asia Pac Fam Med 2011;10:1.

13. Lucas GN. Management of dengue haemorrhagic fever (DHF). Sri Lanka J Child Health 2009;29:107-8.

14. Biswas A, Pangtey G, Devgan V, Singla P, Murthy P, Dhariwal AC, et al. Indian national guidelines for clinical management of dengue fever. J Indian Med Assoc 2015;113:197-206.

15. Gupta E, Ballani N. Current perspectives on the spread of dengue in
India. Infect Drug Resist 2014;7:337.

16. Gupta N, Srivastava S, Jain A, Chaturvedi U. Dengue in India. Indian J Med Res 2012;136:373-90.

17. Yacoub S, Wertheim H, Simmons CP, Screaton G, Wills B. Cardiovascular manifestations of the emerging dengue pandemic. Nat Rev Cardiol 2014;11:335.

18. Nimmannitya S. Management of dengue and dengue haemorrhagic fever. In: Monograph on Dengue/Dengue Haemorrhagic Fever. New Delhi, India: World Health Organization; 1993. p. 55-61.

19. Nhan NT, Phuong CX, Kneen R, Wills B, Van My N, Phuong NT, et al. Acute management of dengue shock syndrome: A randomized doubleblind comparison of 4 intravenous fluid regimens in the first hour. Clin Infect Dis 2001;32:204-13.

20. Idrees S, Ashfaq UA. A brief review on dengue molecular virology, diagnosis, treatment and prevalence in Pakistan. Genet Vaccines Ther 2012;10:6

21. Lye DC, Lee VJ, Sun Y, Leo YS. Lack of efficacy of prophylactic platelet transfusion for severe thrombocytopenia in adults with acute uncomplicated dengue infection. Clin Infect Dis 2009;48:1262-5.

22. Sharma A, Charles K, Chadee D, Teelucksingh S. Dengue hemorrhagic fever in Trinidad and Tobago: A case for a conservative approach to platelet transfusion. Am J Trop Med Hyg 2012;86:531-5.

23. Ooi EE, Gubler DJ. Dengue in Southeast Asia: Epidemiological characteristics and strategic challenges in disease prevention. Cad Saude Publica 2009;25:S115-24.

24. Elsinga J, Lizarazo EF, Vincenti MF, Schmidt M, Velasco-Salas ZI, Arias L, et al. Health seeking behaviour and treatment intentions of dengue and fever: A household survey of children and adults in Venezuela. PLoS Negl Trop Dis 2015;9:e0004237.

25. Deen J, von Seidlein L. Paracetamol for dengue fever: No benefit and potential harm? Lancet Glob Health 2019;7:e552-3.

26. Moore N, Pollack C, Butkerait P. Adverse drug reactions and drug-drug interactions with over the counter NSAIDs. Ther Clin Risk Manage 2015;11:1061.

27. Guha-Sapir D, Schimmer B. Dengue fever: New paradigms for a changing epidemiology. Emerg Themes Epidemiol 2005;2:1.

28. Singhi S, Kissoon N, Bansal A. Dengue and dengue hemorrhagic fever: Management issues in an intensive care unit. J Pediatr (Rio J) 2007;83 Suppl 2:S22-35.

29. Hasan S, Jamdar SF, Alalowi M, Al Beaiji SM. Dengue virus: A global human threat: Review of literature. J Int Soc Prev Community Dent 2016;6:1.

30. Thisyakorn U, Thisyakorn C. Latest developments and future directions in dengue vaccines. Ther Adv Vaccines 2014;2:3-9.

31. Ghosh A, Dar L. Dengue vaccines: Challenges, development, current status and prospects. Indian J Med Microbiol 2015;33:3.

32. Ahamed SF, Rosario V, Britto C, Dias M, Nayak K, Chandele A, et al. Emergence of new genotypes and lineages of dengue viruses during the 2012-15 epidemics in southern India. Int J Infect Dis 2019;84:S34-43.

33. Innis BL, Eckels KH. Progress in development of a live-attenuated, tetravalent dengue virus vaccine by the United States army medical research and materiel command. Am J Trop Med Hyg 2003;69 Suppl 6:1-4

34. Thomas SJ, Yoon IK. A review of Dengvaxia: Development to deployment. Hum Vaccin Immunother 2019;15:2295-314.

35. Bhamarapravati N, Sutee Y. Live attenuated tetravalent dengue vaccine. Vaccine 2000;18:44-7.

36. Guy B, Lang J, Saville M, Jackson N. Vaccination against dengue: Challenges and current developments. Ann Rev Med 2016;67:387-404.

37. Beaumier CM, Gillespie PM, Hotez PJ, Bottazzi ME. New vaccines for neglected parasitic diseases and dengue. Transl Res 2013;162:144-55.

38. Vannice KS, Roehrig JT, Hombach J. Next generation dengue vaccines: A review of the preclinical development pipeline. Vaccine 2015;33:7091-9.

39. Putnak R, Barvir DA, Burrous JM, Dubois DR, D’Andrea VM, Hoke $\mathrm{CH}$, et al. Development of a purified, inactivated, dengue- 2 virus vaccine prototype in Vero cells: Immunogenicity and protection in mice and rhesus monkeys. J Infect Dis 1996;174:1176-84.

40. Raviprakash K, Porter KR, Kochel TJ, Ewing D, Simmons M, Phillips I, et al. Dengue virus Type 1 DNA vaccine induces protective immune responses in rhesus macaques. J Gen Virol 2000;81:1659-67.

41. Fernandez S, Thomas SJ, De La Barrera R, Im-Erbsin R, Jarman RG, et al. An adjuvanted, tetravalent dengue virus purified inactivated vaccine candidate induces long-lasting and protective antibody responses against dengue challenge in rhesus macaques. Am J Trop Med Hyg 2015;92:698-708.

42. Chaturvedi UC, Shrivastava R, Nagar R. Dengue vaccines: Problems 
and prospects. Indian J Med Res 2005;121:639.

43. Qin E, Shi H, Tang L, Wang C, Chang G, Ding Z, et al. Immunogenicity and protective efficacy in monkeys of purified inactivated Vero-cell SARS vaccine. Vaccine 2006;24:1028-34.

44. Danko JR, Beckett CG, Porter KR. Development of dengue DNA vaccines. Vaccine 2011;29:7261-6.

45. Porter KR, Kochel TJ, Wu SJ, Raviprakash K, Phillips I, Hayes CG. Protective efficacy of a dengue 2 DNA vaccine in mice and the effect of $\mathrm{CpG}$ immuno-stimulatory motifs on antibody responses. Arch Virol 1998;143:997-1003.

46. Konishi E, Yamaoka M, Kurane I, Mason PW. A DNA vaccine expressing dengue Type 2 virus premembrane and envelope genes induces neutralizing antibody and memory B cells in mice. Vaccine 2000;18:1133-9.

47. Konishi E, Kosugi S, Imoto JI. Dengue tetravalent DNA vaccine inducing neutralizing antibody and anamnestic responses to four serotypes in mice. Vaccine 2006;24:2200-7.

48. Guy B, Nougarede N, Begue S, Sanchez V, Souag N, Carre M, et al. Cellmediated immunity induced by chimeric tetravalent dengue vaccine in naive or Flavivirus-primed subjects. Vaccine 2008;26:5712-21.

49. Abdullah AA, Lee YK, Chin SP, Lim SK, Lee VS, Othman R, et al. Discovery of dengue virus inhibitors. Curr Med Chem 2020;19:45-8.

50. Halstead SB. Dengavaxia sensitizes seronegative to vaccine enhanced disease regardless of age. Vaccine 2017;35:6355-8.

51. Aguiar M, Stollenwerk N. Dengavaxia: Age as surrogate for serostatus. Lancet Infect Dis 2018;18:245.

52. Turner HC, Wills BA, Rahman M, Cuong HQ, Thwaites GE, Boni MF, et al. Projected costs associated with school-based screening to inform deployment of Dengvaxia: Vietnam as a case study. Trans R Soc Trop Med Hyg 2018;112:369-77.

53. Galula JU, Salem GM, Chang GJ, Chao DY. Does structurally mature dengue virion matter in vaccine preparation in post-Dengavaxia era. Hum Vaccin Immunother 2019;15:2328-36

54. Aguiar M, Halstead SB, Stollenwerk N. Consider stopping dengvaxia administration without immunological screening.

55. Pasteur S. World's First Dengue Vaccine, Approved in Mexico. Philippines: Dengavaxia; 2017

56. Guy B, Almond JW. Towards a dengue vaccine: Progress to date and remaining challenges. Comp Immunol Microbiol Infect Dis 2008;31:239-52.

57. Thomas SJ. Developing a dengue vaccine: Progress and future challenges. Ann N Y Acad Sci 2014;1323:140-59.

58. Koh HL, Teh SY, Noordin NM, Sulaiman LH. Pharmacoeconomic evaluation for dengue vaccine pricing for Malaysia: Towards affordability, cost-effectiveness and sustainability. Asian J Pharm Clin Res 2017;10:12-5.

59. Alim A, Sarfraz A. Dengue vaccine: A road to dengue prevention. Int J Contemporary Med Res 2018;5:C6-9.

60. Murray NE, Quam MB, Wilder-Smith A. Epidemiology of dengue: Past, present and future prospects. Clin Epidemiol 2013;5:299.

61. Shepard DS, Halasa YA, Tyagi BK, Adhish SV, Nandan D, Karthiga KS, et al, INCLEN Study Group. Economic and disease burden of dengue illness in India. Am J Trop Med Hyg 2014;91:1235-42.

62. Zhu G, Liu T, Xiao J, Zhang B, Song T, Zhang Y, et al. Effects of human mobility, temperature and mosquito control on the spatiotemporal transmission of dengue. Sci Total Environ 2019;651:969-78.

63. Promprou S, Jaroensutasinee M, Jaroensutasinee K. Climatic Factors Affecting Dengue Haemorrhagic Fever Incidence in Southern Thailand.2005;29:41-8.

64. Xu L, Stige LC, Chan KS, Zhou J, Yang J, Sang S, et al. Climate variation drives dengue dynamics. P Natl Acad Sci U S A 2017;114:113-8.

65. Banu S, Hu W, Hurst C, Tong S. Dengue transmission in the AsiaPacific region: Impact of climate change and socio-environmental factors. Trop Med Int Health 2011;16:598-607.

66. Naish S, Dale P, Mackenzie JS, McBride J, Mengersen K, Tong S. Climate change and dengue: A critical and systematic review of quantitative modelling approaches. BMC Infect Dis 2014;14:167.

67. Semenza JC, Sudre B, Miniota J, Rossi M, Hu W, Kossowsky D, et al. International dispersal of dengue through air travel: Importation risk for Europe. PLoS Negl Trop Dis 2014;8:e3278.

68. Tambo E, Chen JH, Zhou XN, Khater EI. Outwitting dengue threat and epidemics resurgence in Asia-Pacific countries: Strengthening integrated dengue surveillance, monitoring and response systems. Infect Dis Poverty 2016;5:56.

69. Stoddard ST, Forshey BM, Morrison AC, Paz-Soldan VA, VazquezProkopec GM, Astete H, et al. House-to-house human movement drives dengue virus transmission. Proc Natl Acad Sci U S A 2013;110:994-9.
70. Kyle JL, Harris E. Global spread and persistence of dengue. Ann Rev Microbiol 2008;62:71-92.

71. Rezza G. Aedes albopictus and the reemergence of dengue. BMC Public Health 2012;12:72.

72. Struchiner CJ, Rocklöv J, Wilder-Smith A, Massad E. Increasing dengue incidence in Singapore over the past 40 years: Population growth, climate and mobility. PLoS One 2015;10:e0136286.

73. Kendall C, Hudelson P, Leontsini E, Winch P, Lloyd L, Cruz F. Urbanization, dengue, and the health transition: Anthropological contributions to international health. Med Anthropol Q 1991;5:257-68.

74. Akram DS, Ahmed S. Dengue fever. Infect Dis J 2005;14:124-5.

75. Chen WJ, Chen SL, Chien LJ, Chen CC, King CC, Harn MR, et al. Silent transmission of the dengue virus in Southern Taiwan. Am J Trop Med Hyg 1996;55:12-6.

76. Recker M, Vannice K, Hombach J, Jit M, Simmons CP. Assessing dengue vaccination impact: Model challenges and future directions. Vaccine 2016;34:4461-5

77. Tuan NM, Nhan HT, Chau NV, Hung NT, Tuan HM, Van Tram T, et al. Sensitivity and specificity of a novel classifier for the early diagnosis of dengue. PLoS Negl Trop Dis 2015;9:e0003638.

78. World Health Organization. Dengue Vector Management: Report of a Regional Workshop Colombo, Sri Lanka. Geneva: World Health Organization; 2014.

79. Tapia-Conyer R, Méndez-Galván J, Burciaga-Zúñiga P. Community participation in the prevention and control of dengue: The patio limpio strategy in Mexico. J Paediatr Child Health 2012;32 Suppl 1:10-3.

80. Guo YH, Lai SJ, Liu XB, Li GC, Yu HJ, Liu QY. Governmental supervision and rapid detection on dengue vectors: An important role for dengue control in China. Acta Trop 2016;156:17-21

81. Tapia-Conyer R, Betancourt-Cravioto M, Mendez-Galvan J. Dengue: An escalating public health problem in Latin America. J Paediatr Child Health 2012;32 Suppl 1:14-7.

82. Rafikov M, Rafikova E, Yang HM. Optimization of the Aedes aegypti control strategies for integrated vector management. J Appl Math 2015;2015:1-8.

83. World Health Organization. Integrated Vector Management to Control Malaria and Lymphatic Filariasis: WHO Position Statement. Geneva: World Health Organization; 2011.

84. Singh A, Taylor-Robinson AW. Vector control interventions to prevent dengue: Current situation and strategies for future improvements to management of Aedes in India. J Infect Dis Pathol 2017;2:2.

85. Naranjo DP, Qualls WA, Jurado H, Perez JC, Xue RD, Gomez E, et al. Vector control programs in Saint Johns county, Florida and Guayas, Ecuador: Successes and barriers to integrated vector management. BMC Public Health 2014;14:674.

86. Framework WG. Integrated Vector Management. Geneva, Switzerland: World Health Organization; 2004.

87. Sepulveda LS, Vasilieva O. Optimal control approach to dengue reduction and prevention in Cali, Colombia. Math Methods Appl Sci 2016;39:5475-96.

88. Lizzi KM, Qualls WA, Brown SC, Beier JC. Expanding integrated vector management to promote healthy environments. Trends Parasitol 2014;30:394-400.

89. Chanda E, Ameneshewa B, Bagayoko M, Govere JM, Macdonald MB. Harnessing integrated vector management for enhanced disease prevention. Trends Parasitol 2017;33:30-41

90. Berg HV, Mutero CM, Ichimori K, World Health Organization. Guidance on policymaking for integrated vector management; 2012;1-11.

91. Mitchell-Foster K, Ma BO, Warsame-Ali S, Logan C, Rau ME, Lowenberger C. The influence of larval density, food stress, and parasitism on the bionomics of the dengue vector Aedes aegypti (Diptera: Culicidae): Implications for integrated vector management. J Vector Ecol 2012;37:221-9.

92. Mann RS, Kaufman PE. Natural product pesticides: Their development, delivery and use against insect vectors. Mini Rev Org Chem 2012;9:185-202

93. Bharati M, Saha D. Multiple insecticide resistance mechanisms in primary dengue vector, Aedes aegypti (Linn.) from dengue endemic districts of Sub-Himalayan West Bengal, India. PLoS One 2018;13:e0203207.

94. Marques AM, Kaplan MA. Active metabolites of the genus piper against Aedes aegypti: Natural alternative sources for dengue vector control. Univ Sci 2015;20:61-82.

95. Kalaivani K, Senthil-Nathan S, Murugesan AG. Biological activity of selected Lamiaceae and Zingiberaceae plant essential oils against the dengue vector Aedes aegypti L. (Diptera: Culicidae). Parasitol Res 2012;110:1261-8. 
96. Beula JM, Ravikumar S, Ali MS. Mosquito larvicidal efficacy of seaweed extracts against dengue vector of Aedes aegypti. Asian Pac J Trop Biomed 2011;1:S143-6.

97. Kalimuthu K, Lin SM, Tseng LC, Murugan K, Hwang JS. Bio-efficacy potential of seaweed Gracilaria firma with copepod, Megacyclops formosanus for the control larvae of dengue vector Aedes aegypti. Hydrobiologia 2014;741:113-23.

98. Bond JG, Marina CF, Williams T. The naturally derived insecticide spinosad is highly toxic to Aedes and Anopheles mosquito larvae. Med Vet Entomol Entomol 2004;18:50-6.

99. Subramaniam J, Kovendan K, Kumar PM, Murugan K, Walton W. Mosquito larvicidal activity of Aloe vera (Family: Liliaceae) leaf extract and Bacillus sphaericus, against chikungunya vector, Aedes aegypti. Saudi J Biol Sci 2012;19:503-9.
100. Kumar PM, Murugan K, Kovendan K, Panneerselvam C, Kumar KP, Amerasan $\mathrm{D}$, et al. Mosquitocidal activity of Solanum xanthocarpum fruit extract and copepod Mesocyclops thermocyclopoides for the control of dengue vector Aedes aegypti. Parasitol Res 2012;111:609-18.

101. Sarwar MB. Elimination of dengue by control of Aedes vector mosquitoes (Diptera: Culicidae) utilizing copepods (Copepoda: Cyclopidae). IJBBE 2015;1:53-8

102. Warikoo R, Kumar S. Impact of Argemone mexicana extracts on the cidal, morphological, and behavioral response of dengue vector, Aedes aegypti L. (Diptera: Culicidae). Parasitol Res 2013;112:3477-84.

103.Paulraj MG, Kumar PS, Ignacimuthu S, Sukumaran D. Natural insecticides from actinomycetes and other microbes for vector mosquito control. In: Herbal Insecticides, Repellents and Biomedicines: Effectiveness and Commercialization. New Delhi: Springer; 2016. p. 85-99. 